\title{
An efficient improved particle swarm optimization based on prey behavior of fish schooling
}

\author{
Xiaohu YAN*,**, Fazhi HE*, Yilin CHEN* and Zhiyong YUAN* \\ *School of Computer, Wuhan University \\ Luo-jia-shan, Wuchang, Wuhan, Hubei Province, PRChina, 430072 \\ E-mail: fzhe@whu.edu.cn \\ **Wuhan NARI Limited Company of State Grid Electric Power Research Institute \\ No.143, Luoyu Road, Hongshan District, Wuhan 430074, China
}

Received 30 December 2014

\begin{abstract}
Particle swarm optimization (PSO) is a population based optimization technique to search approximate solutions for extremely difficult or impossible numeric problems. However, PSO may easily get trapped in a local optimum when solving complex multimodal problems. This paper proposes an efficient improved PSO based on prey behavior of fish schooling (FSPSO). Firstly, the fish find better place to prey according to the heuristic water wave which other fish provide, and the heuristic information can be used to enhance the global search ability in PSO. Therefore, the global best particle searches randomly towards several best positions that other particles have experienced. Secondly, the weak fish that cannot move quickly are eaten when the fish schooling are attacked. The decimation of the weak fish can be simulated in PSO to avoid the local optimum and to increase the diversity of the population. Hence, the particles in the immediate vicinity of the global worst particle are replaced by new particles. Finally, the proposed FSPSO is tested on both several standard benchmark functions and a typical NP-complete problem. The results demonstrate that our algorithm achieves remarkable improvements over the basic PSO and even other improved PSOs.
\end{abstract}

Key words : Improved particle swarm optimization, Fish schooling, Prey behavior, Global search ability, Local optimum

\section{Introduction}

The particle swarm optimization is a parallel evolutionary computation technique developed by Kennedy and Eberhart based on the social behavior of bird flocking (Kennedy and Eberhart, 1995; Tang, et al., 2014). PSO is very popular due to its simplicity of implementation and ability to quickly converge to a reasonably good solution. Therefore, PSO is applied to a wide range of problems, including logistics optimization (Yoshiaki, et al., 2014), fault diagnosis (Zhu, et al., 2014), parameter optimization (Marko, et al., 2014; Duan and Hao, 2014), graphics, image, CAD (Wen, et al., 2015; Qi, 2014; Chang, et al., 2014; Huang, et al., 2011; Liu, et al., 2011; Li, et al., 2013; Cheng, et al., 2013), and flowshop scheduling problems (Zhang, et al., 2008; Liao, et al., 2007)

However, PSO may easily get trapped in a local optimum when solving complex multimodal problems (Liang, et al., 2006). Many attempts have been made to improve the performance of PSO, such as inertia weight is changed with iterations, adjusted neighborhood is searched and other search techniques are combined with PSO (Angeline, 1998; Liu, et al., 2005). Eberhart found a significant improvement in the performance with a linearly varying inertia weight over the generations (Hu and Eberhart, 2002). The randomized inertia weight, nonlinear inertia weight (Chatterjee and Siarry, 2006) and dynamically changing inertia weight (Yang, et al., 2007) were proposed. Kennedy claimed that PSO with a small neighborhood might perform better on complex problems, while PSO with a large neighborhood would perform better on simple problems (Kennedy, 1999). Suganthan applied a dynamically adjusted neighborhood where the neighborhood of a particle gradually increased until it included all particles (Suganthan, 1999). 
To enhance the performance, the improved PSOs that are combined with other search techniques have been studied by some researchers (Shelokar, et al., 2007). Evolutionary operators such as selection, crossover, and mutation have been introduced to the PSO to keep the best particles (Shi, et al., 2003). The swarm is divided into subpopulations, and a breeding operator is used within a subpopulation or between the subpopulations to increase the diversity of the population (Lovbjerg, et al., 2001). Negative entropy is used to discourage premature convergence (Xie, et al., 2002). PSO combined with chaos is proposed (Alatas, et al., 2009; Jiang and Bompard, 2005). Higashi and Iba present PSO with gaussian mutation (Higashi and Iba, 2003). Wang and Li integrate PSO and simulated annealing to improve the performance of PSO (Wang and Li, 2004). Hieu and Hiroshi propose a new PSO using differential evolution (DE) with genetic algorithm (GA) to reduce calculation cost and to improve convergence towards the optimal solution (Hieu and Hiroshi, 2013).

The paper proposes an improved particle swarm optimization based on prey behavior of fish schooling, which is different from the social behavior in many references (Neshat, et al., 2012; Yao, et al., 2010). The motivation behind our algorithm is the intelligent prey behavior of fish schooling. The fish find better place to prey according to the heuristic water wave provided by other fish. To imitate the behavior, the global best particle searches randomly towards several best positions that other particles have experienced. The weak fish that cannot move quickly are eaten when the fish schooling are attacked. To simulate the behavior, the particles in the immediate vicinity of the global worst particle are replaced by new particles.

This paper is organized as follows. In Section 2, FSPSO is presented and described. In Section 3, the performance of FSPSO is analyzed by experiments. Finally, conclusions are summarized in Section 4.

\section{Improved particle swarm optimization based on prey behavior of fish schooling}

\subsection{Basic particle swarm optimization}

In basic PSO, the $i$ th particle has a current position $X_{i}$ and a current velocity $V_{i}$, then:

$$
\begin{aligned}
X_{i} & =\left(X_{i 1}, \cdots, X_{i d}, \cdots, X_{i D}\right) \\
V_{i} & =\left(V_{i 1}, \cdots, V_{i d}, \cdots, V_{i D}\right)
\end{aligned}
$$

where $D$ is the search space dimension, $1 \leq i \leq n, 1 \leq d \leq D . P_{i}=\left(P_{i 1}, P_{i 2}, \cdots, P_{i D}\right)$ is the best position that the $i$ th particle have experienced. $P_{g}=\left(P_{g 1}, P_{g 2}, \cdots, P_{g D}\right)$ is the global best position among all the particles (Liu, et al., 2010). Then the evolution equation of PSO is:

$$
\begin{gathered}
V_{i d}^{k+1}=w V_{i d}^{k}+c_{1} r_{1}\left(P_{i d}^{k}-X_{i d}^{k}\right)+c_{2} r_{2}\left(P_{g d}^{k}-X_{i d}^{k}\right) \\
X_{i d}^{k+1}=X_{i d}^{k}+V_{i d}^{k+1}
\end{gathered}
$$

where $w$ is inertia weight, $c_{1}$ and $c_{2}$ are learning coefficients, $r_{1}$ and $r_{2}$ are two separately generated uniformly distributed random numbers in the range [0,1], $k$ is the iteration number. The first part of Eq. (3) represents the previous velocity, which provides the necessary momentum for particles to roam across the search space. The second part, known as the cognitive component, represents the personal thinking of each particle. The third part is known as the social component, which represents the collaborative effect of the particles, in finding the global optimal solution (Ratnaweera, et al., 2004).

\subsection{Prey behavior of fish schooling}

It has been investigated that complex pressure waves and other water movements are used by fish schooling as communication methods in prey behavior (Larsson, 2009). The fish will find better place to prey according to the heuristic water wave. Therefore, the water wave can be viewed as heuristic information in PSO. In the prey process, the fish schooling will be attacked by other predators. In the darwinian world of fish schooling, the weak fish that cannot move quickly will be eaten (Ota, et al., 2003). The survival of the fittest is the law of nature (Patel, 2002). Hence, elimination of the weak particles in PSO can improve the the fitness of the population.

In this paper, FSPSO simulates the prey behavior of fish schooling to improve the performance. To enhance the global search ability, the global best particle searches randomly towards several best positions that other particles have experienced. To avoid the local optimum and to increase the diversity of the population, the particles in the immediate vicinity of the global worst particle are replaced by random particles. 


\subsection{Improve the global best position}

The fish find better place to prey according to the heuristic information provided by other fish. To imitate the behavior in PSO, the global best particle should search to new directions according to the heuristic information that $m$ other particles provide, hoping that the search processing will lead to find better position than the global best one.

Without loss of generality, the $m$ particles are chosen randomly, and the best positions that the $m$ particles have experienced are $P_{1}, P_{2}, \cdots, P_{m}$. Then the directions that the global best particle searches are shown in Fig. 1.

Since we select the new directions from a limited set specialized by the best positions of the $m$ particles, our algorithm is different from local search method, which will search the neighbour space.

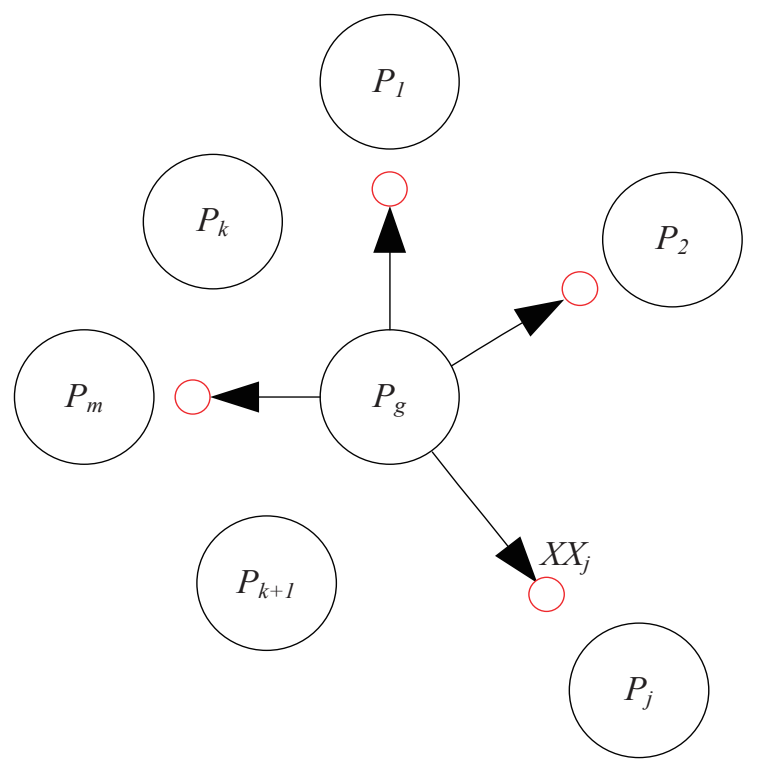

Fig. 1 Directions the global best particle searches

As shown in Fig. 1, the global best particle searches randomly towards several best positions that the $m$ particles have experienced, and $P_{1}, P_{2}, P_{j}, P_{m}$ are the positions that the global best particle searches towards. Then the position of the $j$ th direction that the global best particle searches is as follows:

$$
X X_{j}=P_{g}+r_{3} \bullet c_{3} \bullet\left(P_{j}-P_{g}\right)
$$

where $1 \leq j \leq n, r_{3}$ is a random number uniformly distributed in the range $[0,1], c_{3}$ is the search coefficient. If the best position in $X X_{j}$ is better than $P_{g}, P_{g}$ is replaced by the best position. Then the global search ability of FSPSO can be enhanced.

\subsection{Improve particles in the immediate vicinity of the global worst particle}

The weak fish that cannot move quickly are eaten when the fish schooling are attacked. To simulate the behavior, the weak particles are replaced by new particles that are generated randomly. In our algorithm, the particles in the immediate vicinity of the global worst particle are the weak particles.

Without loss of generality, the position of the global worst particle is $P_{b}=\left(P_{b 1}, P_{b 2}, \cdots, P_{b D}\right)$, the position of the $j$ th weak particle is $Y_{j}$, and the distance between $Y_{j}$ and $P_{b}$ is short. Then the relationship of $Y_{j}$ and $P_{b}$ is shown in Fig. 2.

As shown in Fig. 2, $Y_{1}, Y_{2}, Y_{j}$ are the weak particles, and they are in the circle, then:

$$
\sum_{i=1}^{D}\left|Y_{j i}-P_{b i}\right| \leq c_{4} \bullet \text { Range }
$$

where Range is the search range of particles, $c_{4}$ is the range coefficient. The weak particles in the immediate vicinity of $P_{b}$ are replaced by random particles. Therefore, the diversity of the population is increased and FSPSO can avoid the local optimum. 


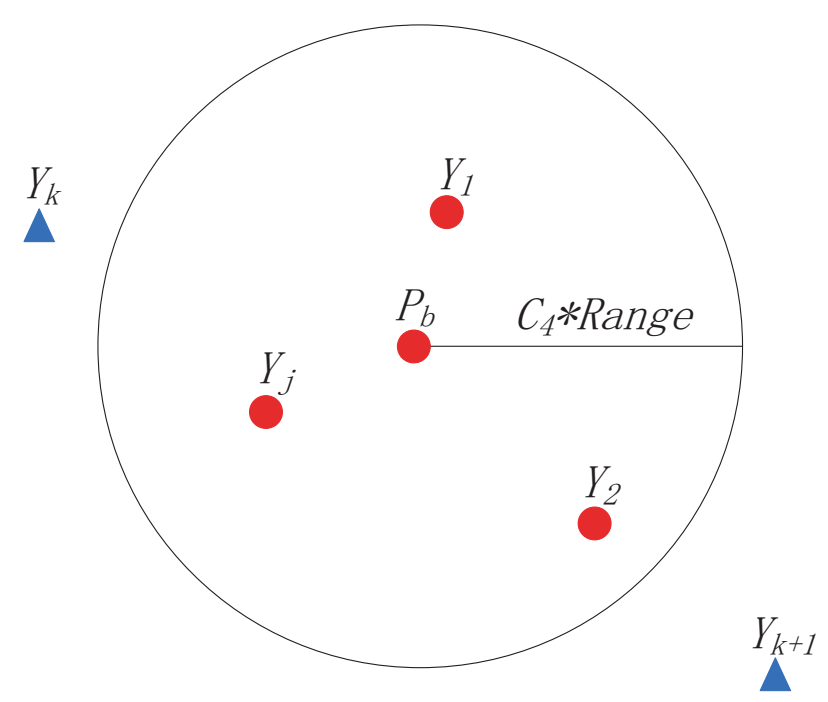

Fig. 2 The particles in the immediate vicinity of the global worst particle

\subsection{The step of FSPSO}

FSPSO that simulates the prey behavior of fish schooling is proposed. The steps of FSPSO are as follows:

Step 1: The parameters of FSPSO are initialized. The individual number $n$, the maximum iteration number maxk, inertia weight $w$, learning coefficients $c_{1}$ and $c_{2}$, search coefficient $c_{3}$ and range coefficient $c_{4}$ are initialized.

Step 2: The position and velocity of the particle are updated according to Eq. (3) and Eq. (4).

Step 3: The global best position is improved according to Eq. (5).

Step 4: The particles in the immediate vicinity of the global worst particle are improved according to Eq. (6).

Step 5: If the termination condition of FSPSO is satisfied, output the position of the global best particle which is the solution of the optimization problem; otherwise, turn to Step 2.

\subsection{The complexity analysis of FSPSO}

In FSPSO, the maximum iteration number is maxk. It takes $O(n * D)$ to compute the position of each particle's own personal best position $P_{i}$, the global best position $P_{g}$ and the global worst position $P_{b}$. It takes $O(n * D)$ to replace the particles in the immediate vicinity of the global worst particle with random particles. The time complexity that the global best particle searches better position is $O(m)$. As $m$ is small, then the time complexity of FSPSO is $O(n * D * \operatorname{maxk})$. In FSPSO, $P_{i}, P_{g}$ and $P_{b}$ should be preserved. Then the space complexity of FSPSO is $O(n * D)$. Therefore, the space and time complexity of FSPSO are the same as those of PSO, and the performance of FSPSO is high.

\section{The analysis of experiment}

\subsection{Function optimization}

In order to evaluate the performance of FSPSO, the algorithm is applied to optimize several standard benchmark functions (Van den Bergh and Engelbrecht, 2004; Rashedi, et al., 2009; Li, et al., 2013; Eckart, et al., 2000). The experiment environment is as follows: CPU: 17-2600k @3.4GHz; physical memory: 8GB; software: Matlab 2013b. The benchmark functions are in Table 1.

Table 1 Benchmark functions

\begin{tabular}{|l|l|}
\hline \multicolumn{1}{|c|}{ Function } & \multicolumn{1}{c|}{ Range } \\
\hline$f_{1}(x)=\sum_{i=1}^{N} x_{i}^{2}$ & {$[-\mathbf{1 0 0 , 1 0 0}]$} \\
\hline$f_{2}(x)=\sum_{i=1}^{N}\left|x_{i}\right|+\prod_{i=1}^{N}\left|x_{i}\right|$ & {$[-\mathbf{1 0 , 1 0}]$} \\
\hline$f_{3}(x)=\sum_{i=1}^{N}\left(\sum_{j=1}^{j=i} x_{j}\right)^{2}$ & {$[-\mathbf{1 0 0 , 1 0 0}]$} \\
\hline$f_{4}(x)=\max _{i}\left\{\left|x_{i}\right|, 1 \leq i \leq N\right\}$ & {$[-\mathbf{1 0 0 , 1 0 0}]$} \\
\hline$f_{5}(x)=\sum_{i=1}^{N-1}\left(100\left(x_{i+1}-x_{i}^{2}\right)^{2}+\left(x_{i}-1\right)^{2}\right)$ & {$[-\mathbf{3 0 , 3 0}]$} \\
\hline$f_{6}(x)=\sum_{i=1}^{N}\left(\left\lfloor x_{i}+0.5\right\rfloor\right)^{2}$ & {$[-\mathbf{1 0 0 , 1 0 0}]$} \\
\hline$f_{7}(x)=\sum_{i=1}^{N}\left(x_{i}^{2}-10 \cos \left(2 \pi x_{i}\right)+10\right)$ & {$[-\mathbf{5 . 1 2 , 5 . 1 2}]$} \\
\hline$f_{8}(x)=-20 \exp \left(-0.2 \sqrt{\frac{1}{N} \sum_{i=1}^{N} x_{i}^{2}}\right)-\exp \left(\frac{1}{N} \sum_{i=1}^{N} \cos \left(2 \pi x_{i}\right)\right)+20+e$ & {$[-32,32]$} \\
\hline$f_{9}(x)=\sum_{i=1}^{N} \frac{x_{i}^{2}}{4000}-\prod_{i=1}^{N} \cos \left(\frac{x_{i}}{\operatorname{sqrti}}\right)$ & {$[-\mathbf{6 0 0 , 6 0 0}]$} \\
\hline
\end{tabular}


$f_{1}(x)-f_{6}(x)$ are unimodal functions and $f_{7}(x)-f_{9}(x)$ are multimodal functions. In these functions, $N$ is the dimension of the function, the minimum value $(\mathrm{fmin})$ of the functions are zero, and the optimum location $\left(X_{o p t}\right)$ for functions are in $[0]^{N}$. FSPSO is applied to optimize these functions as well as PSO and GA. Then the results are compared to evaluate the performance of FSPSO. In the three algorithms, the dimension is $30(N=D=30)$, population is 50 and maximum iteration number is 1000. In PSO, $c_{1}=c_{2}=2$, inertia factor $(w)$ is decreasing from 0.9 to 0.7 linearly with the iterations. Compared experiments are made to find the appropriate values of parameters in FSPSO and the results are as follows: $c_{3}=1.5, c_{4}=0.0001, m=6$, and other parameters are set as the same as PSO. The parameters of GA are set as follows: single point uniform crossover with the rate of 0.95 , random selection mechanism, gaussian mutation with the rate of 0.1 (Karaboga and Basturk, 2008). All experiments run 30 times. The best, average, median and variance values of the solutions obtained by GA, PSO and FSPSO are given in Table 2.

Table 2 Function optimization results obtained by the GA, PSO and FSPSO

\begin{tabular}{|l|l|l|l|l|l|}
\hline Function & Algorithm & Best & Average & Median & Variance \\
\hline \multirow{5}{*}{$f_{1}(x)$} & GA & 0.14592 & 0.63625 & 0.58375 & 0.41057 \\
\cline { 2 - 6 } & PSO & 0.002427 & 2.7357 & 0.68436 & 3.6599 \\
\cline { 2 - 6 } & FSPSO & 0.002104 & 2.1229 & 0.57377 & 2.946 \\
\hline \multirow{5}{*}{$f_{2}(x)$} & GA & 1.2841 & 3.2947 & 3.2745 & 1.4063 \\
\cline { 2 - 6 } & PSO & 0.019645 & 0.73631 & 0.6005 & 0.69595 \\
\cline { 2 - 6 } & FSPSO & 0.006164 & 0.56639 & 0.32647 & 0.63668 \\
\hline \multirow{5}{*}{$f_{3}(x)$} & GA & 1.1849 & 3.8748 & 3.2517 & 1.8998 \\
\cline { 2 - 6 } & PSO & 0.94601 & 1100.9 & 623.68 & 1562.3 \\
\cline { 2 - 6 } & FSPSO & 0.006776 & 407.19 & 135.98 & 613.01 \\
\hline \multirow{5}{*}{$f_{5}(x)$} & GA & 0.57362 & 0.71238 & 0.71985 & 0.094686 \\
\cline { 2 - 6 } & PSO & 0.01335 & 0.18487 & 0.17502 & 0.14434 \\
\cline { 2 - 6 } & FSPSO & 0.012606 & 0.17555 & 0.13542 & 0.08965 \\
\hline \multirow{3}{*}{$f_{6}(x)$} & GA & 0.43722 & 100.49 & 112.73 & 51.818 \\
\cline { 2 - 6 } & PSO & 0.000707 & 17.702 & 9.7506 & 19.366 \\
\cline { 2 - 6 } & FSPSO & 0.000623 & 8.8467 & 3.3622 & 10.234 \\
\hline \multirow{3}{*}{$f_{7}(x)$} & GA & 0.081631 & 1.1758 & 0.68473 & 2.0158 \\
\cline { 2 - 6 } & PSO & 0.001161 & 1.0023 & 0.14625 & 1.6907 \\
\cline { 2 - 6 } & FSPSO & 0.000173 & 1.0008 & 0.1364 & 1.6649 \\
\hline \multirow{5}{*}{$f_{8}(x)$} & GA & 2.8672 & 12.977 & 11.281 & 7.313 \\
\cline { 2 - 6 } & PSO & 0.000286 & 0.48013 & 0.096227 & 0.8008 \\
\cline { 2 - 6 } & FSPSO & 0.000107 & 0.42118 & 0.12176 & 1.2556 \\
\hline \multirow{3}{*}{$f_{9}(x)$} & GA & 0.28697 & 1.2358 & 1.2599 & 0.42428 \\
\cline { 2 - 6 } & PSO & 0.034773 & 3.2062 & 3.4702 & 2.3946 \\
\cline { 2 - 6 } & FSPSO & 0.000107 & 2.8061 & 3.3161 & 1.9283 \\
\hline & GA & 0.029616 & 0.063848 & 0.058647 & 0.022878 \\
\cline { 2 - 6 } & PSO & 0.01908 & 0.61927 & 0.57628 & 0.38699 \\
\cline { 2 - 6 } & FSPSO & 0.013158 & 0.04613 & 0.03821 & 0.01732 \\
\hline & & & & & \\
\hline
\end{tabular}

As shown in Table 2, the best values obtained by FSPSO are better than those obtained by GA and PSO for all functions. Then the global search ability of FSPSO is better. The average values obtained by FSPSO are better than those obtained by PSO for all functions, and the average values obtained by FSPSO are better than those obtained by GA besides $f_{1}(x), f_{3}(x)$ and $f_{8}(x)$. Then the whole search ability of FSPSO is better. The median values obtained by FSPSO are smaller than those obtained by PSO besides $f_{7}(x)$, and the median values obtained by FSPSO are smaller than those obtained by GA besides $f_{3}(x)$ and $f_{8}(x)$. The variance values obtained by FSPSO are smaller than those obtained by PSO except for $f_{7}(x)$, and the variance values obtained by FSPSO are smaller than those obtained by GA except for $f_{1}(x), f_{3}(x)$ and $f_{8}(x)$. Then the stability of FSPSO is better.

The best values of the solutions obtained by the three algorithms for benchmark functions are compared in Fig. 3. To compare distinctly, the best values are taken logarithm.

As shown in Fig. 3, the best values of the solutions obtained by FSPSO is smaller than those obtained by other algorithms, and the global search ability is stronger. The convergence curves are used to evaluate the performance of GA, PSO and FSPSO. A random unimodal function $f_{2}(x)$ and a random multimodal function $f_{7}(x)$ are chosen to be optimized by these algorithms. To analyze the convergence curves better, the fitness values in the iteration are taken logarithm. The convergence curves of the three algorithms for the functions are shown in Fig. 4 and Fig. 5.

According to these figures, FSPSO has better search ability and convergence for the two functions. FSPSO tends to find the global optimum faster and hence has a higher convergence rate. Compared to PSO, FSPSO is more efficient. Therefore, FSPSO shows remarkable improvements to optimize the benchmark functions. 


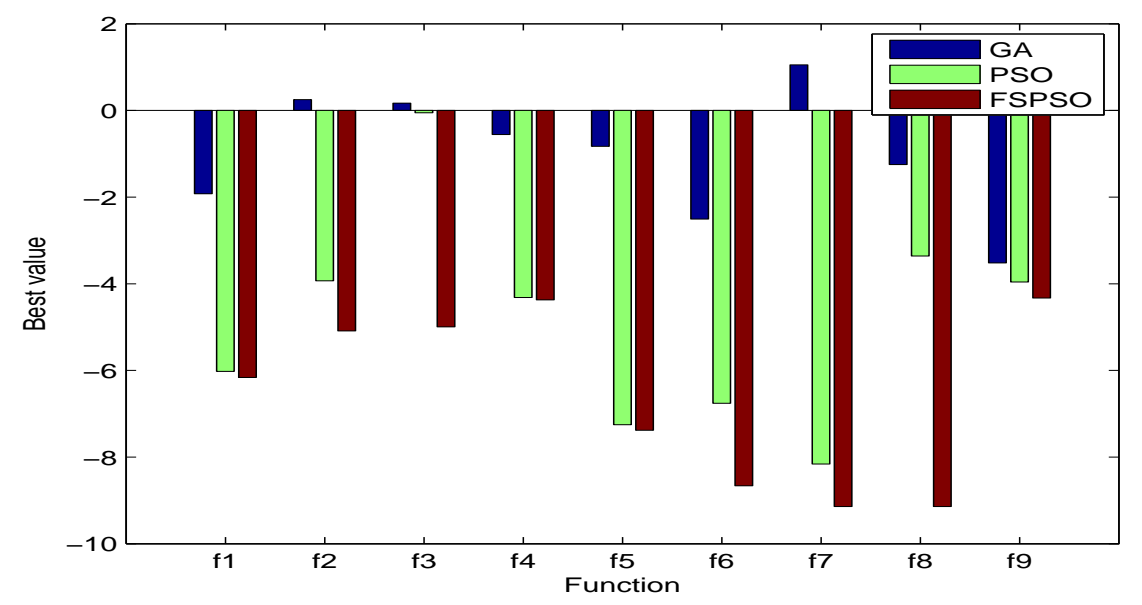

Fig. 3 Best values for benchmark functions

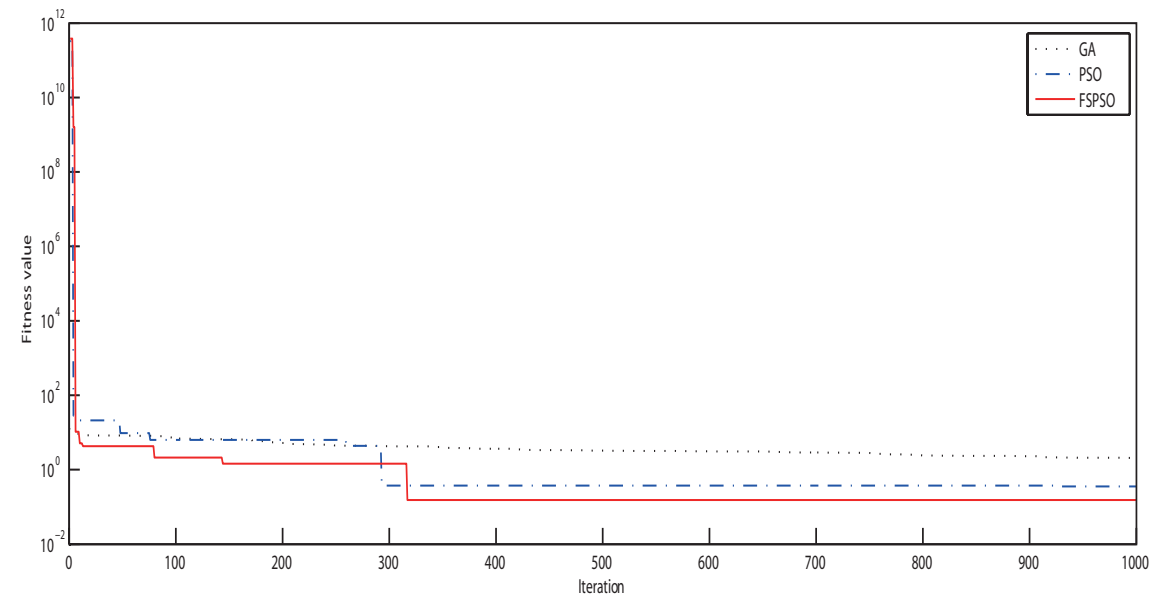

Fig. 4 Convergence curves for $f_{2}(x)$

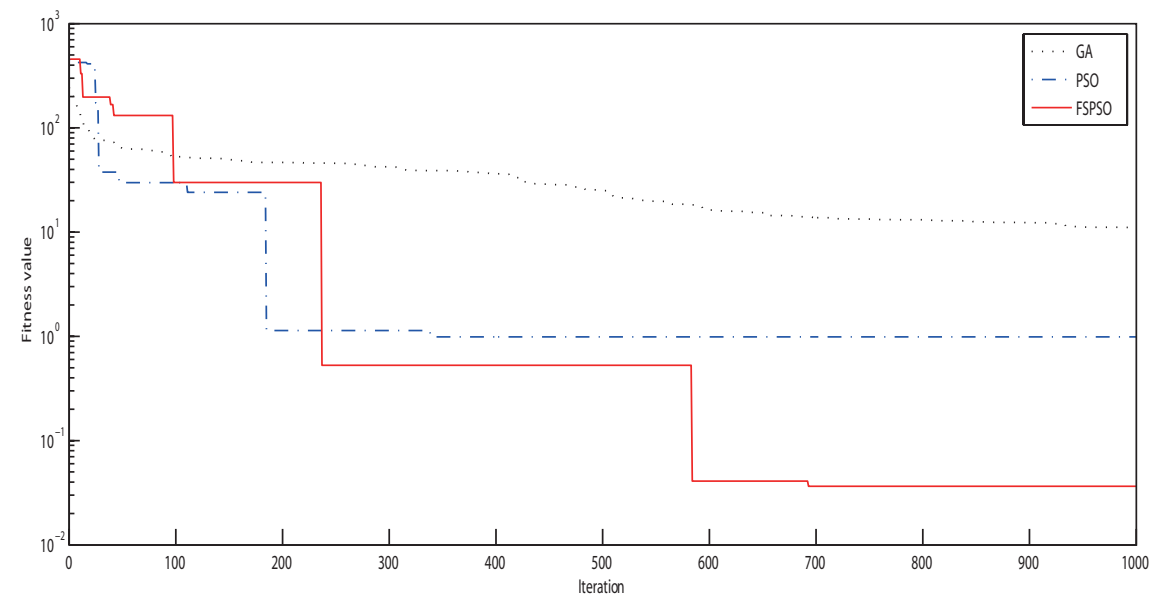

Fig. 5 Convergence curves for $f_{7}(x)$ 


\subsection{Knapsack problem}

The knapsack problem is a typical NP-complete problem. The GA, PSO and FSPSO are used to optimize a knapsack problem (Sinha and Zoltners, 1979; Martello, et al., 2000; Chen and Ross, 2014; Chu and Beasley, 1998; Zhao and Li, 2014). The parameters of three algorithms and the experiment environment are the same as those in function optimization. The value matrix $C$ and volume matrix $W$ are as follows:

$$
\begin{gathered}
\mathrm{C}=\left(\begin{array}{l}
220208198192180180165162160158 \\
155130125122120118115110105101 \\
1001009896959088828077 \\
75737270696665636058 \\
5650302015108531
\end{array}\right) \\
W=\left(\begin{array}{l}
80828570727066505525 \\
50554048503222603032 \\
40383532252830225030 \\
45306050206520253010 \\
2025151010104421
\end{array}\right)
\end{gathered}
$$

The limited volume is 1000. As knapsack problem is a discrete problem, then the position of each particle $i$ is defined as:

$$
X_{i d}^{k}= \begin{cases}1 & \text { if } r_{4}<\operatorname{sig}\left(V_{i d}^{k}\right) \\ 0 & \text { else }\end{cases}
$$

where $\operatorname{sig}\left(V_{i d}^{k}\right)=\frac{1}{1+\exp \left(-V_{i d}^{k}\right)}, r_{4}$ is a random number uniformly distributed in the range $[0,1]$. The convergence curves of the three algorithms for the knapsack problem are in Fig. 6.

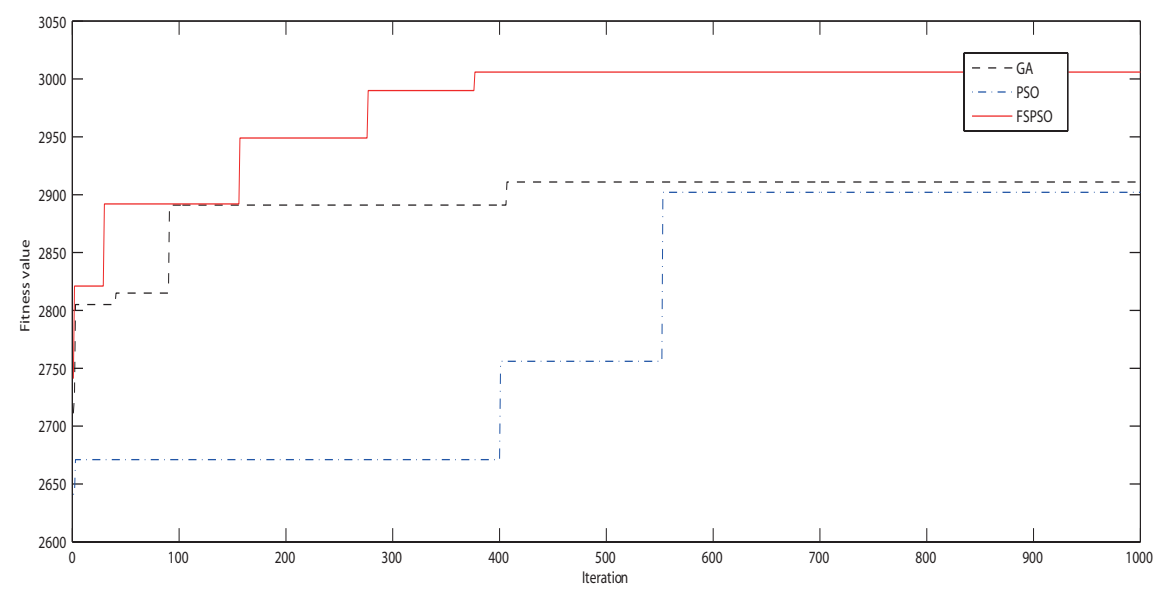

Fig. 6 Convergence curves for the knapsack problem

As shown in Fig. 6, the global best value of FSPSO is greater than those obtained by other algorithms, and the global search ability is stronger. The three algorithms run 30 times to optimize the knapsack problem. The best, worst, average, median and variance values of the best solutions obtained by the GA, PSO and FSPSO are given in Table 3.

Table 3 Knapsack problem optimization results obtained by the GA, PSO and FSPSO

\begin{tabular}{|c|c|c|c|c|c|}
\hline Algorithm & Best & Worst & Average & Median & Variance \\
\hline GA & 2909 & 2670 & 2788.5 & 2787 & 49.5 \\
\hline PSO & 2955 & 2687 & 2820.3 & 2812 & 58.7 \\
\hline FSPSO & 3030 & 2899 & 2957.4 & 2961 & 31.8 \\
\hline
\end{tabular}

As shown in Table 3, the best, worst, average and median values obtained by the FSPSO are greater than the corresponding values obtained by the GA and PSO, and the variance value obtained by the FSPSO is smaller. Thus, the global search ability and convergence of the FSPSO are better than those of the other two algorithms, indicating that the FSPSO is more efficient for solving the knapsack problem. 


\subsection{Further analysis of FSPSO in function optimization}

The main concepts of FSPSO are improvement of the global best position and elimination of the weak particles. Then their performances are compared individually to optimize the benchmark function $f_{1}(x)$. Algorthm1 is PSO combined with improvement of the global best position and Algorthm2 is PSO combined with elimination of the weak particles.

We also compare our algorithm with other improved PSOs, particularly with the PSO using improved global best position, such as the improved PSO combined with local search (Wu, 2014), which is named LSPSO in this paper. The comparison results are in Fig. 7, and the fitness values are taken logarithm

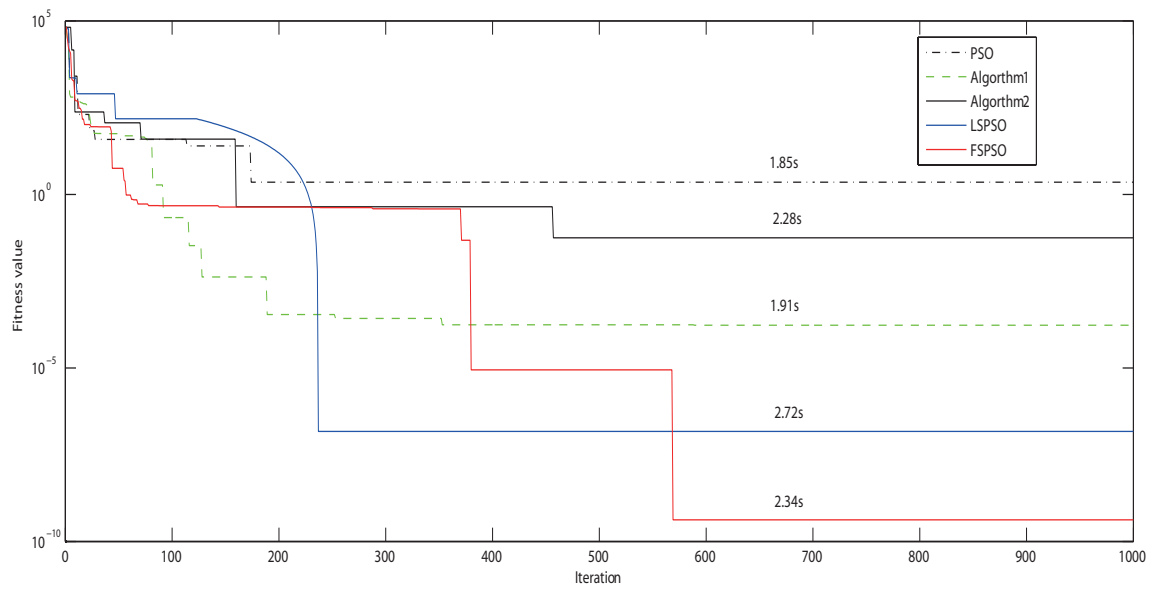

Fig. 7 Further analysis of FSPSO

As shown in Fig. 7, the solution quality of both Algorthm1 and Algorthm2 are improved compared to basic PSO. So, the individual effectiveness of our idea, improvement of the global best position and elimination of the weak particles, are confirmed. And the solution quality of FSPSO is the best one among the five algorithms.

The runtimes of PSO, Algorthm1, Algorthm2, LSPSO and FSPSO are 1.85, 2.28, 1.91, 2.72 and 2.34 seconds, respectively. In future work, we will extend our FSPSO in parallel computation architecture to reduce computation time while keep the solution quality.

According to Fig. 7, the runtime of FSPSO is little longer than that of basic PSO. However, the runtime difference is acceptable when considering that the solution quality of FSPSO is much better than that of basic PSO.

According to Fig. 7, both the solution quality and runtime of FSPSO are better than those of LSPSO, so our FSPSO can outperform other improved PSOs.

\section{Conclusion}

An efficient improved particle swarm optimization based on prey behavior of fish schooling is presented in the paper. The global best particle searches randomly towards several best positions that other particles have experienced, therefore the global search ability becomes stronger in FSPSO. The particles in the immediate vicinity of the global worst particle are replaced by random particles, therefore the diversity of the population is increased and the local optimum is efficiently avoided in FSPSO. The proposed algorithm has been tested to optimize several standard benchmark functions and a typical NP-complete problem. The obtained results confirm the high performance of FSPSO in solving the continuous and discrete optimization problems. In future work, we will extend FSPSO in both parallel computing methods such as multi-core CPU/GPU/APU architectures and applications such as collaborative design of embedded hardware/software partition.

\section{Acknowledgement}

The presented research work is supported by the National Basic Research Program of China (973 Program) (Grant No. 2011CB707904) and the National Natural Science Foundation of China (Grant No. 61472289). Corresponding author, fzhe@whu.edu.cn (Fazhi He). 


\section{References}

Alatas, B., Akin, E. and Ozer, A.B., Chaos embedded particle swarm optimization algorithms, Chaos Solitons \& Fractals, Vol.40, No.4 (2009), pp.1715-1734.

Angeline, P.J., Using selection to improve particle swarm optimization, Proceedings of the IEEE Congress on Evolutionary Computation (1998), pp.84-89.

Chang, B.M., Tsai, H.H. and Shih, J.S., Using fuzzy logic and particle swarm optimization to design a decision-based filter for cDNA microarray image restoration, Engineering Applications of Artificial Intelligence, Vol.36 (2014), pp.12-26.

Chatterjee, A. and Siarry, P., Nonlinear inertia weight variation for dynamic adaptation in particle swarm optimization,Computers \& Operations Research, Vol.33, No.3 (2006), pp.859-871.

Chen, K. and Ross, S.M., An adaptive stochastic knapsack problem, European Journal of Operational Research, Vol. 239, No.3 (2014), pp.625-635.

Cheng, Y., He, F.Z., Cai, X.T. and Zhang, D.J., A group undo/redo method in 3D collaborative modeling systems with performance evaluation, Journal of Network and Computer Applications, Vol.36, No.6 (2013), pp.1512-1522.

Chu, P.C. and Beasley, J.E., A genetic algorithm for the multidimensional knapsack problem, Journal of Heuristics, Vol.4, No.1 (1998), pp.63-86.

Duan, C.Z. and Hao, Q.L., Surface roughness prediction of end milling process based on IPSO-LSSVM, Journal of Advanced Mechanical Design Systems and Manufacturing, Vol.8, No.3 (2014), DOI: 10.1299/jamdsm.2014jamdsm0024.

Eckart, Z., Kalyanmoy, D. and Lothar, T., Comparison of multiobjective evolutionary algorithms: empirical results, Evolutionary Computation, Vol.8, No.2 (2000), pp.173-195.

Hieu, P. and Hiroshi, H., Adaptive plan system of swarm intelligent using differential evolution with genetic algorithm, Journal of Advanced Mechanical Design Systems and Manufacturing, Vol.7, No.3 (2013), pp.458-473.

Higashi, N. and Iba, H., Particle swarm optimization with gaussian mutation, Proceedings of the IEEE Swarm Intelligence Symposium (2003), pp.72-79.

Huang, Z.Y., He, F.Z., Cai, X.T., Zou, Z.Q., Liu, J., Liang, M.M. and Chen, X., Efficient random saliency map detection, Science China Information Sciences, Vol.54, No.6(2011), pp.1207-1217.

$\mathrm{Hu}$, X.H. and Eberhart, R., Multiobjective optimization using dynamic neighborhood particle swarm optimization, Proceedings of the IEEE Congress on Evolutionary Computation (2002), pp.1677-1681.

Jiang, C.W. and Bompard, E., A hybrid method of chaotic particle swarm optimization and linear interior for reactive power optimisation, Mathematics and Computers in Simulation, Vol.68, No.1 (2005), pp.57-65.

Karaboga, D. and Basturk, B., On the performance of artificial bee colony (ABC) algorithm, Applied Soft Computing, Vol.8, No.1 (2008), pp.687-697.

Kennedy, J., Small worlds and mega-minds: Effects of neighborhood topology on particle swarm performance, Proceedings of the Congress on Evolutionary Computation (1999), pp.1931-1938.

Kennedy, J. and Eberhart, R.C., Particle swarm optimization, IEEE International Conference on Neural Networks (1995), pp.1942-1948.

Larsson, M., Possible functions of the octavolateralis system in fish schooling, Fish and Fisheries, Vol.10, No.3(2009), pp.344-353.

Liang, J.J., Qin, A.K., and Baskar, S., Comprehensive learning particle swarm optimizer for global optimization of multimodal functions, IEEE Transactions on Evolutionary Computation, Vol.10, No.3 (2006), pp.281-295.

Liao, C.J., Tseng, C.T. and Luarn, P., A discrete version of particle swarm optimization for flowshop scheduling problems, Computers \& Operations Research, Vol.34, No.10 (2007), pp.3099-3111.

Liu, B., Wang, L., Jin, Y.H., Tang, F. and Huang, D.X., Improved particle swarm optimization combined with chaos, Chaos Solitons \& Fractals, Vol.25, No.5 (2005), pp.1261-1271.

Liu, C.A., Yan, X.H., Liu, C.Y. and Li, G.D., Dynamic path planning for mobile robot based on improved genetic algorithm, Chinese Journal of Electronics, Vol.19, No.2 (2010), pp.245-248.

Liu, H.J., He, F.Z., Cai, X.T., Chen, X. and Chen, Z., Performance-based control interfaces using mixture of factor analyzers, The Visual Computer, Vol.27, No.6-8 (2011), pp.595-603.

Li, X.X., He, F.Z., Cai, X.T., Zhang, D.J. and Chen, Y.L., A method for topological entity matching in the integration of heterogeneous cad systems, Integrated Computer-Aided Engineering, Vol.20, No.1 (2013), pp.15-30. 
Li, Y.Q., Chi, Y.H. and Wen, T., A dynamic boundary based particle swarm optimization, Acta Electronic Sinica, Vol.41, No.5 (2013), pp.865-870.

Lovbjerg, M., Rasmussen, T.K. and Krink, T., Hybrid particle swarm optimizer with breeding and subpopulations, Genetic and Evolutionary Computation Conference (2001), pp.469-476.

Marko, H., Simon, K., Tomaz, I., Matej, P., Joze, B. and Miran, B., Turning parameters optimization using particle swarm optimization, Procedia Engineering, Vol.69 (2014), pp.670-677.

Martello, S., Pisinger, D. and Toth, P., New trends in exact algorithms for the 0-1 knapsack problem, European Journal of Operational Research, Vol.123, No.2 (2000), pp.325-332.

Neshat, M., Sepidnam, G., Sargolzaei, M. and Toosi, A.N., Artificial fish swarm algorithm: a survey of the state-ofthe-art, hybridization, combinatorial and indicative applications, Artificial Intelligence Review, Vol.42, No.4 (2014), pp.965-997.

Ota, T., Nguyen, T.A., Huang, E., Detrich, HW. and Amemiya, CT.,Positive darwinian selection operating on the immunoglobulin heavy chain of antarctic fishes, Journal of Experimental Zoology Part B-Molecular and Developmental Evolution, Vol.295B, No.1 (2003), pp.45-58.

Patel, A., Survival of the fittest and zero sum games, Fluctuation and Noise Letters, Vol.2, No.4 (2002), pp. L279-L284.

Qi, C.M., Maximum entropy for image segmentation based on an adaptive particle swarm optimization, Applied Mathematics \& Information Sciences, Vol.8, No.6 (2014), pp.3129-3135.

Rashedi, E., Nezamabadi-pour, H. and Saryazdi, S., GSA: a gravitational search algorithm, Information Sciences, Vol.179 (2009), pp.2232-2248.

Ratnaweera, A., Halgamuge, S. and Watson, H.C., Self-organizing hierarchical particle swarm optimizer with timevarying acceleration coefficients, IEEE Transactions on Evolutionary Computation, Vol.8, No.3 (2004), pp.240-255.

Shelokar, P.S., Siarry, P., Jayaraman, V.K. and Kulkarni, B.D., Particle swarm and ant colony algorithms hybridized for improved continuous optimization, Applied Mathematics and Computation, Vol. 188, No.1 (2007),pp.129-142.

Shi, X.H., Lu, Y.H., Zhou, C.G., Lee, H.P., Lid, W.Z.and Liang, Y.C., Hybrid evolutionary algorithms based on PSO and GA, Proceedings of IEEE Congress on Evolutionary Computation (2003), pp.2393-2399.

Suganthan, P.N., Particle swarm optimizer with neighborhood operator, Proceedings of the IEEE Congress on Evolutionary Computation (1999), pp.1958-1962.

Tang, D.Y., Cai, Y.M., Zhao, J. and Xue, Y., A quantum-behaved particle swarm optimization with memetic algorithm and memory for continuous non-linear large scale problems, Information Sciences, Vol.289, No.24 (2014), pp.162-189.

Van den Bergh, F. and Engelbrecht, A.P., A cooperative approach to particle swarm optimization, IEEE Transactions on Evolutionary Computation, Vol.8, No.3 (2004), pp.225-239.

Wang, X.H. and Li, J.J., Hybrid particle swarm optimization with simulated annealing, Proceedings of the Third International Conference on Machine Learning and Cybernetics (2004), pp.2402-2405.

Wen, L., Wang, X.C., Wu, Z.K., Zhou, M.Q. and Jin, J.S., A novel statistical cerebrovascular segmentation algorithm with particle swarm optimization, Neurocomputing, Vol.148 (2015), pp.569-577.

Wu, G.H., Qiu, D.S., Yu, Y., Pedrycz, W., Ma, M. and Li, H.F., Superior solution guided particle swarm optimization combined with local search techniques, Expert Systems with Applications, Vol.41, No.16 (2014), pp. 7536-7548.

Xie, X.F., Zhang, W.J. and Yang, Z.L., A dissipative particle swarm optimization, Proceedings of IEEE Congress on Evolutionary Computation (2002), pp.1456-1461.

Yang, X.M., Yuan, J.S., Yuan, J.Y. and Mao, H.N., A modified particle swarm optimizer with dynamic adaptation, Applied Mathematics and Computation, Vol.189 (2007), pp.1205-1213.

Yao, X.G., Zhou, Y.Q. and Li, Y.M., Hybrid algorithm with artificial fish swarm algorithm and PSO,Application Research of Computers, Vol.27, No.6 (2010), pp.2084-2086.

Yoshiaki, S., Tatsuhiko, S. and Takatobu, M., Parallel computing for huge scale logistics optimization through binary PSO associated with topological comparison, Journal of Advanced Mechanical Design Systems and Manufacturing, Vol.8, No.1 (2014), DOI:10.1299/jamdsm.2014jamdsm0005.

Zhang, C.S., Sun, J.G., Zhu, X.J. and Yang, Q.Y., An improved particle swarm optimization algorithm for flowshop scheduling problem, Information Processing Letters, Vol.108, No.4 (2008), pp.204-209.

Zhao, C.X. and Li, X.Y., Approximation algorithms on 0-1 linear knapsack problem with a single continuous variable, Journal of Combinatorial Optimization, Vol.28, No.4 (2014), pp.910-916.

Zhu, K.H., Song, X.G. and Xue, D.X., A roller bearing fault diagnosis method based on hierarchical entropy and support vector machine with particle swarm optimization algorithm, Measurement, Vol.47 (2014), pp.669-675. 